\title{
¿Son útiles los anticoagulantes orales en el tratamiento de los pacientes con enfermedad coronaria?
}

Oral anticoagulation therapy in patients with coronary artery disease: a meta-analysis. Anand SS, Yusuf S. JAMA 1999; 282: 2058-2067.

\section{Objetivo}

Determinar los efectos de la anticoagulación oral a largo plazo, estratificado por rango de intensidad (valores de RIN) y uso concomitante de aspirina, sobre la incidencia de muerte, reinfarto, ACV y sangrado en pacientes con enfermedad coronaria establecida.

\section{Fuente y selección de datos}

Se seleccionaron a través de una búsqueda en MEDLINE, EMBASE, CURRENT CONTENTS, listas de referencias y datos aportados por expertos y compañías farmacéuticas aquellos estudios randomizados que incluyeron pacientes con enfermedad coronaria, tratados con anticoagulación oral por lo menos 3 meses, publicados entre 1960 y julio de 1999. De 43 artículos seleccionados fueron analizados 31 ensayos.

\section{Resultados principales}

La anticoagulación de intensidad alta (RIN 2.8-4.8) en comparación con controles en 10.056 pacientes produjo una reducción clara de la mortalidad (Odds Reduction \{0Rde\} 22\% (IC95\% 13 a 31\%), infarto ORde 42\% (IC 95\% 34 a 48\%) y complicaciones tromboembólicas incluyendo ACV, 0Rde $63 \%$ (IC 95\% 53 a 71\%) pero se asoció con un incremento de 6.0 veces el riesgo de sangrado mayor (IC 95\% 4.4 a 8.2 veces). La anticoagulación de rango moderado (RIN 2-3) versus control en 1365 pacientes, el OR para mortalidad fue de 18\% (IC 95\% 6 a
$37 \%$ ), para infarto $52 \%$ (IC 95\% 37 a $64 \%$ ) y para ACV $53 \%$ (IC $95 \% 19$ a $73 \%$ ) pero con un incremento del sangrado en 7.7 veces (IC $95 \% 3.3$ a 18 veces). La intensidad moderada a alta de anticoagulación (RIN $\geq 2$ ) versus aspirina en 3457 pacientes no mostró una reducción de la mortalidad, infarto o ACV y estuvo asociada a un incremento de 2.4 veces (IC $95 \% 1.6$ a 3.6 veces) de sangrado mayor. Para la anticoagulación moderada a alta más aspirina versus aspirina sola en 480 pacientes el OR para mortalidad, infarto o ACV fue de $56 \%$ (IC $95 \% 17$ a 77 con un incremento del sangrado mayor de 1.9 veces (IC 95\% 0.6 a 6.0 veces). Para la anticoagulación de baja intensidad (RIN <2) más aspirina versus aspirina sola en 8435 pacientes no hubo una reducción significativa en la mortalidad, infarto o ACV y se evidenció un incremento del sangrado mayor en 1.3 veces (IC $95 \% 1.0$ a 1.8 veces)

\section{Conclusiones}

La anticoagulación oral de intensidad moderada y alta es efectiva en La reducción de la incidencia de infarto y ACV pero incrementa el riesgo de sangrado. En presencia de aspirina, la anticoagulación de baja intensidad no parece ser superior a la aspirina sola, mientras que la anticoagulación de moderada a alta intensidad (RIN 2-3) más aspirina parece ser más efectiva que aspirina sola en la prevención de eventos con solo un modesto incremento del riesgo de sangrado.

\section{COMENTARIO}

La significativa tasa de recurrencia de eventos vasculares y la persistencia de la actividad trombótica por períodos prolongados en los pacientes que han sufrido un evento coronario agudo, a pesar del uso de aspirina, ha sido el motivo del interés en el uso de los anticoagulante orales como una alternativa en el tratamiento de este cuadro ${ }^{1 .}$ La eficacia demostrada en los estudios con estrategias combinadas sostiene la hipótesis de un mecanismo independiente de beneficio en la conjunción de las terapéuticas antiplaquetaria y antitrombótica pero esto aún no ha sido totalmente confirmado?

Los resultados del actual metaanálisis demuestran que en pacientes con enfermedad coronaria la anticoagulación oral de moderada a alta intensidad junto con aspirina, es superior a la aspirina sola en la reducción de eventos vasculares pero a un costo de casi 2 veces más riesgo de sangrado mayor. Surge una pregunta ¿es suficientemente importante el beneficio demostrado por los anticoagulantes orales, para pagar el altísimo y - dramático - costo de esta tasa de eventos hemorrágicos mayores?. La evaluación de los resultados clínicos en estudios randomizados frecuentemente representan el ideal y esto puede no reflejarse en la práctica diaria ${ }^{3}$. El uso de los anticoagulantes orales muchas veces se ve dificultado por el inconveniente de un monitoreo estricto y un ajuste periódico de la dosis que afecta la adherencia del paciente sumado al riesgo potencial de las complicaciones hemorrágicas en pacientes con alta probabilidad de requerir procedimientos invasivos. Además, poseen un estrecho margen terapéutico con el consiguiente riesgo de hemorragia o trombosis que implica una anticoagulación por encima o por debajo de una adecuada ventana terapéutica, respectivamente. El riesgo de sangrado está directamente relacionado a la intensidad de la anticoagulación, a factores clínicos, la duración de la terapia y el uso concomitante de antiplaquetarios. La aspirina es actualmente la droga más costo-efectiva en la prevención secundaria de la enfermedad coronaria. Administrada después de un infarto, la aspirina disminuye la frecuencia de muerte o infarto recurrente en más del $20 \%$. En pacientes con angina inestable la aspirina puede evitar 30\% de casos de infarto en forma segura y barata. También se benefician sustancialmente los pacientes sometidos a cirugía coronaria, angioplastia y los que padecen angina crónica estable 4 .

Cada 3.4 eventos prevenidos por 1000 pacientes tratados, ocurre un sangrado mayor y esta relación es demasiado costosa. El beneficio absoluto de un tratamiento es críticamente dependiente del riesgo basal del paciente individual. Puesto que los efectos colaterales del tratamiento con anticoagulantes orales son sustanciales, el conocimiento del riesgo basal es crítico para la toma de decisiones en el paciente individual y justificar su utilización.

\section{Dr. Pablo F. Oberti}

Servicio de Cardiología. Hospital Italiano de Buenos Aires.

\section{Referencias}

1. Pathogenesis of thrombosis in coronary artery disease. Chesebro JH, Rauch U, Fuster V, Badimon JJ. Haemostasis 1997; 27 : 12-18.

2. Long term oral anticoagulant therapy in patients with unstable angina or suspected non-Q-wave myocardial infarction: organization to assess strategies for ischemic syndromes

(OASIS) pilot study results. Anand SS, Yusuf S, Pogue J, Weitz JI, Flather M. Circulation 1998; 98: 1064-1070.

3. The quality of anticoagulation management. Ansell JE. Arch Intern Med 2000; 160: 895-896.

4. Aspirin, the poor man's statin? Verheugt FWA. Lancet 1998; 351: 227-232. 